\title{
Traduire
}

Une autre perspective sur r tr traduction

Revue française de la traduction

$221 \mid 2009$

Voies de l'interprétation

\section{Entretien avec Gilles Ouvrard, Interprète de conférence}

Freddie Plassard

\section{(2) OpenEdition}

1 Journals

Édition électronique

URL : http://journals.openedition.org/traduire/345

DOI : $10.4000 /$ traduire.345

ISSN : 2272-9992

Éditeur

Société française des traducteurs

Édition imprimée

Date de publication : 15 décembre 2009

Pagination : 39-47

ISSN : 0395-773X

\section{Référence électronique}

Freddie Plassard, «Entretien avec Gilles Ouvrard, Interprète de conférence », Traduire [En ligne], 221 |

2009, mis en ligne le 12 novembre 2013, consulté le 26 novembre 2020. URL : http://

journals.openedition.org/traduire/345; DOI : https://doi.org/10.4000/traduire.345 


\section{Entretien avec Gilles Ouvrard, Interprète de conférence}

\section{Propos recueillis par Freddie Plassard}

\section{CHOIX DE LA PROFESSION}

- Quels sont les critères qui ont motivé votre choix de la langue à nos yeux exotique voire inaccessible qu'est le chinois, ainsi que de cette culture?

Les raisons pour lesquelles j'ai appris le chinois - qui est de moins en moins exotique en France et n'est heureusement pas inaccessible - sont les suivantes. À l'époque ma sœur aînée faisait de l'anglais (elle vit d'ailleurs depuis trente ans aux États-Unis), et je pense que je voulais me démarquer d'elle. J'ai fait un bac scientifique, mais j'étais surtout bon en langues. Et c'est ma mère qui m'a conseillé de faire du chinois. Ce qui est extraordinaire, c'est qu'elle ne connaissait pas du tout ni le chinois ni la Chine, mais elle m'a dit tel quel " Je pense que ça devrait bien te convenir ". Elle ne s'était pas trompée! Je me suis d'emblée passionné pour l'étude du chinois, que j'ai appris aux Langues' 0 , en montant comme on dit à Paris, car je viens de l'Est... de la France. Quant à la culture chinoise, je dois préciser tout de suite une chose : je la connais en réalité assez mal. Je ne suis pas du tout un sinologue, par exemple. J'ai consacré plusieurs années à l'apprentissage de la langue, et ensuite ce sont les études à l'ESIT qui m'ont mobilisé, avant de me donner un et même deux métiers, la traduction et l'interprétation. J'ai mené de front les deux carrières, à titre libéral, pendant plus de vingt-cinq ans. Autrement, l'intuition de ma mère s'est vraiment révélée très profonde, car si je trouve que la Chine - que je connais encore très peu même si j'y suis allé plusieurs fois par an pendant plus de deux décennies - est un pays fascinant mais terrible, je me suis spontanément toujours bien entendu avec les gens que j'y ai rencontrés... Voilà, j'espère que mes motivations ne vous paraissent pas trop futiles!

- Y a-t-il dans votre biographie personnelle, des éléments qui vous prédestinaient au choix de la profession d'interprète?

II faut que je vous avoue quelque chose : j'ai fait l'ESIT par hasard ! C'est un ami chinois des Langues'0 qui m'a suggéré de tenter les examens d'entrée, et il se trouve que j'ai réussi les 
deux. J'ai d'abord fait Traduction, car mon anglais oral était vraiment trop faible. Et surtout ne le répétez à personne, mais ce qui m'a alors intéressé dans I'ESIT, c'était la possibilité de faire du chinois à haut niveau... Tout à fait le contraire de la vocation professionnelle de l'École.

Donc, non, je n'avais pas de vocation particulière pour devenir interprète, contrairement à certains de mes condisciples qui ne rêvaient que de cela depuis l'enfance, pour des raisons familiales par exemple. Moi, ce n'était pas le cas, je n'étais pas fils ni descendant de diplomates, ni de grands voyageurs. La seule frontière que je connaissais étant enfant, c'était celle qui sépare les Ardennes de la Meuse, c'est vous dire. Plus sérieusement, je dois mon début de carrière d'interprète auprès du Quai d'Orsay à un autre hasard, dramatique celui-ci : à l'époque où je terminais mes études, quelques personnes interprétaient pour les missions officielles, par exemple, le spécialiste de la linguistique du chinois Alain Peyraube travaillait pour le Quai aussi, mais c'est Monsieur Patrick Destenay, de I'Université d'Aix-en-Provence, qui était interprète officiel pour le chinois. Or il est décédé brutalement au moment où j'entrais dans la vie professionnelle, et c'est Christopher Thiéry qui a insisté pour que le tout jeune interprète que j'étais parte en 1983 en mission officielle pour le Président Mitterrand, alors que l'Ambassade [de France à Pékin] ne voulait pas de moi car ils ne me connaissaient pas du tout, pour la bonne raison que je n'étais encore jamais allé à Pékin !

J'ai fait cette mission avec une collègue d'origine chinoise, Madame Vizcarra - Zhang Huafang. Par la suite j'ai souvent pensé à Patrick Destenay. Je sais que les autorités chinoises l'appréciaient. Je n'avais jamais eu l'occasion de le rencontrer, mais j'ai pu constater que les étudiants qu'il avait contribué à former avaient tous un excellent chinois. Pour revenir à votre question, j'ai mené de front les deux carrières, à titre libéral, en tirant beaucoup de satisfaction de ce double exercice professionnel, l'un venant en quelque sorte équilibrer l'autre : l'interprétation avec beaucoup de contacts et de déplacements, et une grande " exposition " comme on dit maintenant et du stress, car j'ai fait carrière uniquement comme consécutiviste (j'ai dû faire en tout cinq à six jours de simultanée), la traduction plus calme même s'il y a aussi évidemment des urgences, mais c'est un travail plus « intérieur " si je puis dire. J'ajoute que pour l'interprétation, l'aspect officiel a quantitativement représenté dans ma vie beaucoup moins de travail que le privé, car outre le gouvernement, j'ai, en fait, surtout travaillé pour le privé, les grandes entreprises. Cela a représenté d'ailleurs une autre forme d'équilibre, et une source de grande diversité : je dois aux délégations chinoises d'avoir visité un grand nombre d'installations industrielles fascinantes, tant en France qu'en Chine, et même outre-mer. Et ma première mission en Chine, c'était avec les pétroliers de Total, dans le Sud du pays. Bien sûr, étant interprète pour le gouvernement, j'ai eu la chance de côtoyer des personnalités historiques, et il y a des gens remarquables partout, mais les rencontres qui m'ont marqué, je les dois aussi aux entreprises. 


\section{MOMENTS FORTS DE LA CARRIÈRE}

- Vous avez été interprète du ministère des Affaires Étrangères, quelles étaient les conditions de recrutement lorsque vous avez débuté ? Sont-elles toujours les mêmes?

Si je me souviens bien, ma première mission - j'étais encore à l'École - m'a été payée $300 \mathrm{~F}$. Ce n'était pas royal, même pour un étudiant. Mais les choses ont très vite évolué, grâce au travail de fond qu'avait mené l'AllC, et, au Quai, grâce à Christopher Thiéry. II s'est battu pour une revalorisation de la rémunération, qu'il a réussi à faire pratiquement décupler. Et entre les ministères, les niveaux de rémunération étaient à peu de choses près les mêmes. Et puis c'était l'époque où l'on partait au bout du monde sur un simple coup de fil. La demande, le coup de téléphone reçu, si on était libre, avaient valeur de contrat. Si la mission était annulée du fait du demandeur, l'interprète était payé comme si elle avait eu lieu. La rémunération n'a pas été vraiment remise en cause plus tard, mais en revanche les autres conditions ont changé : en particulier le préavis avant la confirmation d'une mission. Je ne trouvais pas normal, dans les derniers temps (j'ai arrêté ou plutôt suspendu mon activité d'interprète le 7 juillet 2007), qu'à quelques jours seulement d'une mission importante, on soit encore dans l'expectative. Je précise d'ailleurs que ce n'est pas la faute des services recruteurs, mais des directives reçues. Je comprends parfaitement le souci de ne pas dépenser inutilement de l'argent, mais je pense qu'il aurait sans doute été préférable de rediscuter du principe du paiement systématique en cas d'annulation de la demande.

- À l'occasion de la visite d'officiels ou de personnalités, il n'est pas rare de voir les interprètes à l'œuvre. Comment ressentez-vous le fait d'être sous les feux de la rampe?

Vous parlez de la consécutive, à la télévision on aperçoit de temps à autre l'interprète avec les personnalités, ou sur les photos pendant les séances pour la presse. Quand elles étaient petites et qu'il y avait une visite officielle, mes filles me guettaient pendant les actualités. La plaisanterie à la maison c'était : "C'est qui le monsieur à côté de Papa ? ". Mais l'interprète n'oublie jamais que les feux de la rampe ne brillent pas pour lui. Et je peux vous assurer qu'en fait à l'instant " $t$ ", on n'a pas le temps de se poser des questions parce qu'on est concentré sur son travail. Les missions officielles, c'est en réalité un véritable tourbillon, avec de rares pauses, et des moments d'accélération où tout s'enchaîne. L'interprète est un petit rouage dans une grosse machine, et une personne parmi des dizaines d'autres qui doivent trouver leur place à table ou retrouver leur voiture avant que le cortège ne démarre. On marche dans un couloir en interprétant pour les personnalités, on fait attention à ne pas perdre le contact ni être bousculé par les gens qui suivent. Les photos ou les images, on les découvre après, de l'extérieur, avec un œil étranger. J'ai d'ailleurs souvent eu l'impression qu'il s'agissait de quelqu'un d'autre, et dans le fond, c'est vrai, ce n'est pas moi, c'est l'interprète, qui est sur la photo. Aujourd'hui, ce n'est plus moi, mais il y a toujours un interprète. Dans l'instant, la seule chose qui compte, c'est de faire son travail. L'individu s'efface derrière la fonction, la mission. Donc on ressent la pression, mais surtout avant, pas pendant. Quant aux photos, il faut savoir que pour les professionnels, elles devraient toujours être "propres ", ce qui veut dire sans l'interprète dessus ! 
- Quels sont les moments forts, inoubliables de votre carrière?

Les moments forts, évidemment, c'est par exemple mon premier contact avec une délégation chinoise, je me souviens parfaitement m'être dit « Ah c'est ça une délégation, c'est ça interpréter ! „. Ça m'a semblé facile, les gens étaient gentils... Autrement, il y a mon premier voyage à Hong-Kong, dans ma chambre au Peninsula Hotel j'ai pris une photo de moi en peignoir devant la glace, puis dans le Sud de la Chine quand nous nous sommes retrouvés sur le ferry et qu'il fallait se débrouiller parce que de l'autre côté personne ne nous attendait. Monsieur Li Youyuan, carrure impressionnante et voix caverneuse, mon premier pétrolier mandarin chinois, en 1980. Évidemment, en 1983, ma première mission présidentielle en Chine à Pékin avec le Président Mitterrand, dans le Grand Hall du Peuple, le discours pendant le banquet, quand pour accéder à la tribune, le Président montait les marches de son côté et moi du mien, la version chinoise du discours à la main, version manuscrite couverte de ratures et de rajouts. Mais aussi à Nankin, pendant la même mission quand la collègue chinoise dont je ne connais toujours pas le nom aujourd'hui est venue m'aider pour traduire tout le discours du lendemain, fort long et fort littéraire. Des années plus tard, les discussions à propos de poésie ou de l'histoire de Chine, dont le Président Chirac connaissait certains détails mieux que les Chinois présents eux-mêmes. Enfin les circonstances dramatiques entourant en cours de mission le décès de Monsieur Zhang Tong, un des chefs du programme spatial chinois, et au cours desquelles j'ai fait la connaissance de sa famille, notamment de son épouse, Madame Yu Lizhen, l'une des personnes les plus admirables que j'aie connues.

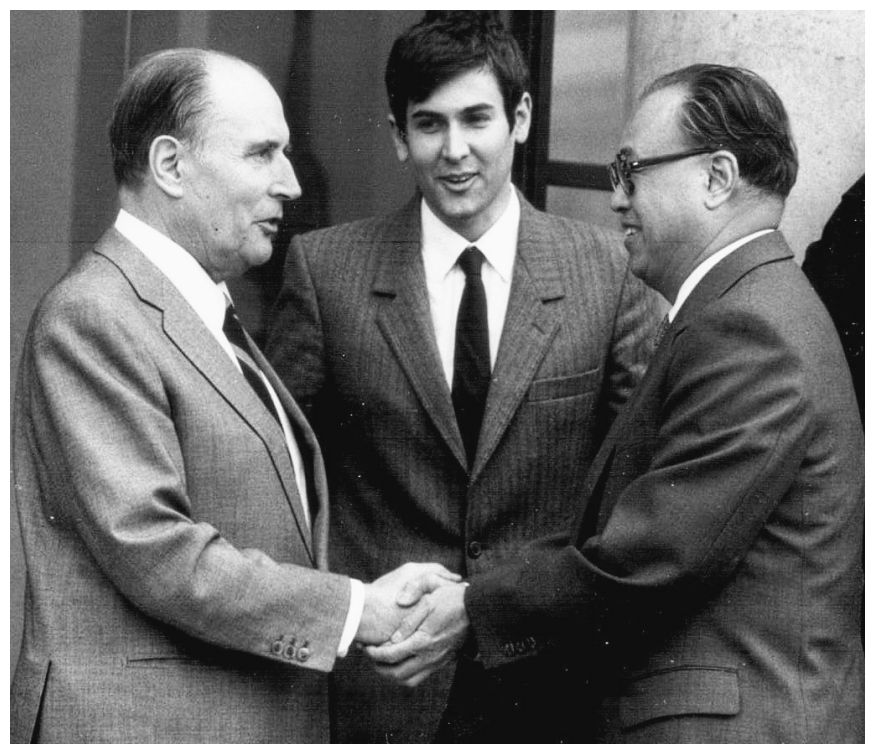

Perron de l'Elysée, 1984 (de gauche à droite)

le Président Mitterrand, G. Ouvrard, Zhao Ziyang, Premier ministre. 
En réfléchissant à votre question, je pense qu'au fond je n'ai rien oublié de toutes les missions que j'ai faites, et qu'au sens propre, d'une certaine façon, aucun moment n'est devenu " oubliable ". Si je reprenais toutes mes notes d'honoraires, par exemple, je crois que je pourrais me remémorer toutes mes missions, et me souvenir de la plupart des gens que j'ai alors rencontrés.

\section{- Qu'est-ce qui donne de l'aisance à un interprète?}

Nous parlons toujours de la consécutive, mais c'est sans doute largement la même chose pour la simultanée. J'ai abordé indirectement ce sujet tout à l'heure, à propos des feux de la rampe. Selon moi, il y a deux éléments : la certitude qu'on a fait le maximum pour se préparer, et la conviction que de toutes façons, "Quand il faut y aller, il faut y aller ! " Je me souviens justement du Président [de l'Assemblée nationale] Jacques Chaban-Delmas disant cela pendant un banquet avant de poser sa serviette pour faire son discours, alors qu'il n'était pas exactement un " bleu ", contrairement à moi. En d'autres termes, c'est le fait de "faire son métier ". C'est alors le stress, le "bon " stress, qui vous donne l'aisance requise. Vous devez avoir de l'aisance, alors vous l'avez. Ou plus précisément, dans un premier temps, vous donnez l'impression de l'avoir, et quand vous constatez que oui, le public vous suit, que ça marche, alors oui, vous l'avez.

- La profession d'interprète est auréolée de prestige. Avez-vous a contrario des souvenirs cuisants?

En fait, le prestige en question est en trompe-l'œil. Le reflet de la lumière qui est braquée sur la personnalité pour laquelle vous traduisez. Être pris en photo ou passer à la télé ne fait pas de vous quelqu'un de prestigieux. Bien faire son travail en cabine, ou derrière son ordinateur, est à mon sens tout aussi prestigieux, même si c'est moins médiatique. Mais pour les souvenirs cuisants, j'en ai un, au début de ma carrière : je trouvais l'orateur à côté de la plaque et même ridicule, et du coup ça m'a déstabilisé et je me suis senti ridicule, et par ailleurs je ne connaissais pas assez le sujet, un épisode de l'histoire de la Longue Marche. Après mon interprétation, laborieuse, Madame Deng Yinchao, qui n'était autre que la veuve du Premier ministre Chou En-Lai (Zhou Enlai), m'a dit gentiment qu'il faudrait que je révise mon histoire et ma géographie ! Et au fait une autre fois aussi, avec des sinologues qui connaissaient beaucoup mieux le sujet que moi, et s'impatientaient à juste titre de mes approximations.

- Qu'est-ce qu'un interprète ne peut à aucun prix se permettre? Qu'est-ce qui le met le plus en difficulté ?

Il y a à nouveau deux choses. La première, sans hésitation, c'est la logistique, et notamment le risque de retard. J'entends encore Monsieur Emmanuel Weintraub nous dire qu'on pouvait tout pardonner à un interprète, sauf d'être mal habillé et d'être en retard. Ensuite par rapport à votre question précédente, je m'aperçois qu'a priori, les souvenirs cuisants que j'ai - même 
s'il y en a heureusement peu - semblent surtout liés à l'impréparation. Comme je l'ai fait remarquer tout dernièrement, à l'occasion de ma première mission à l'étranger en tant que directeur de la section Traduction de l'ESIT, lorsqu'il s'agit d'interpréter pour le chef de l'État, on est content : un d'avoir reçu une bonne formation, deux de s'être bien préparé. Et finalement c'est la même chose : c'est l'image de celui qui vous a embauché, de celui pour lequel vous travaillez, qui est en jeu.

- Vous avez mis un terme à votre carrière d'interprète. Quels sont les aspects de cette profession qui vous manquent, ceux que vous ne regrettez pas?

Stricto sensuj'ai suspendu mon activité, notamment parce que je voyais que j'allais avoir trop de travail pour l'École pour rester suffisamment disponible en libéral, mais je n'ai pas décidé d'y mettre définitivement un terme. Je reprendrai peut-être. Je n'ai pas arrêté parce que j'en avais assez. Bien sûr au bout de plus de vingt-cinq ans, on a un peu le sentiment d'avoir fait le tour de la question, mais dans les dernières années, par exemple, je me disais en revenant de mission que finalement, j'aimais toujours bien ça. Le sentiment d'avoir bien travaillé, d'avoir été utile. Mais je ne peux pas dire que ça me manque, parce que ma vie d'enseignant, et maintenant de directeur, pour quelques années, est très remplie, je vois beaucoup de gens, je fais des choses nouvelles, j'ai beaucoup de travail ; je suis très occupé sur le plan personnel aussi. Ah si, au fait, ce qui me manque, c'est de ne pas avoir eu l'occasion de croiser Madame Carla Bruni! Non, franchement pour le moment, à part le contact avec les collègues pendant les missions, rien ne me manque.

Inversement, si parfois on trouve un peu le temps long, pendant des négociations qui traînent, par exemple, ou bien la compagnie forcée de gens que vous n'appréciez pas énormément ce qui m'est très peu arrivé -, j'ai toujours su que ça faisait partie des inconvénients du métier ; l'avantage c'est que ça ne dure pas très longtemps. Et le gros intérêt de l'exercice libéral, si vous gagnez assez d'argent, c'est la liberté : vous pouvez refuser de travailler pour quelqu'un si vous ne l'appréciez pas, contrairement au salarié qui ne peut pas changer de patron.

- Traductrice, j'ai l'impression de passer ma vie à déchiffrer le monde, ce et ceux qui m'entourent, les codes, les comportements, d'être dans une perpétuelle activité de décryptage. Comment "éprouve-t-on " le monde quand on est interprète?

Je dirais que c'est tout à fait semblable, car on a besoin de repérer tout de suite qui fait quoi, et pourquoi, afin d'être immédiatement efficace. L'interprète, en général, c'est l'instantané, la rapidité de réaction. Et en permanence jauger le vouloir, les vouloirs, de l'émetteur comme du récepteur, pour optimiser la communication. D'où le besoin de se ressourcer, en prenant davantage son temps. 


\section{FORMATION}

\section{Rétrospective}

- Vous êtes issu d'une École dont le renom n'est plus à faire. Après une carrière d'interprète d'une trentaine d'années, quelle est la dette dont vous souhaiteriez vous acquitter auprès de vos maîtres, ou, en d'autres termes, quel a été à vos yeux l'apport essentiel de cette formation ?

C'est une question très intéressante, parce que comme je l'entends, elle est morale, et non pas technique. Je préfère l'idée d'apport essentiel à celle de dette, car je considère qu'un étudiant ne peut pas avoir de dette envers ses maîtres, qu'il rate ses études et change d'orientation, ou les réussisse et puisse gagner sa vie. Chacun est et reste libre, ou plutôt a le droit de se sentir libre, de ne pas se sentir redevable. Les enseignants sont payés pour enseigner, il n'y a pas de dette à cet égard. L'ESIT est une école professionnelle, exigeante, il est normal qu'en en sortant on puisse gagner sa vie. Mais l'apport en ce qui me concerne a été décisif, car il a consisté pour moi à me faire passer du monde des mots, du dictionnaire, des dictionnaires où je me plongeais avec fascination et ravissement pendant des heures, à celui des idées et des choses ou des actions qu'elles désignent et rendent possibles, à ce qui permet de vivre et de faire vivre ceux que l'on aime. C'est capital. Et cela donne une obligation : celle d'essayer d'aider d'autres à suivre ce chemin qui a réussi pour vous pendant une partie importante de votre vie. En clair, d'enseigner à votre tour. C'est l'apport essentiel, et l'obligation qui en découle. Mais au-delà de cela, il y a encore autre chose. La chance d'avoir eu comme professeur quelqu'un comme Danica Seleskovitch. Nos professeurs étaient tous très bons, et de grands professionnels évidemment. Mais Danica, c'était différent. Je lui ai écrit un jour qu'elle nous avait apporté la lumière, je suis persuadé que si vous interrogiez les autres étudiants qui étaient en recherche avec elle, vous obtiendriez la même réponse. Quarante ans plus tard, la limpidité et la justesse de ses écrits continuent à agir, sur des gens qui ne l'ont jamais approchée ni connue. Attention, je ne suis pas en adoration devant une idole. J'aurais des reproches à faire à Mademoiselle Seleskovitch, ses emportements, ses excès, son intransigeance. Je n'ai jamais eu à en pâtir, elle m'avait à la bonne ! C'était le revers de sa générosité et de son enthousiasme. D'autres, comme Marianne Lederer, dont l'intelligence est aussi tranchante que l'était celle de Danica, incarnent pour moi la prudence et la mesure, et les deux sont en fait nécessaires. Mais sans faire de peine à Marianne, je dirai que c'était Danica qui nous galvanisait. "Ne vous contentez pas de bien faire ce que vous savez déjà faire, travaillez aussi et surtout ce que vous ne savez pas bien faire et améliorez-vous ". Son conseil, sa demande instante de l'époque ont gardé pour moi toute leur actualité après quelque trente ans. Elle nous a littéralement tirés vers le haut, et maintenant qu'elle n'est plus là, elle continue à nous pousser aux fesses, si vous me passez l'expression. Et ça n'a rien à voir avec l'interprétation. C'était de la vie dont elle parlait. En ce sens, le mot de maître est effectivement le seul adapté, avec le mot d'élève, au très nombreux pluriel sans doute. Au final, qu'y a-t-il de plus important que d'apprendre à quelqu'un à vivre? 
- Si vous deviez résumer cet apport en un mot, quel serait-il ?

La volonté.

\section{Prospective}

- Les "métiers de l'interprétation ", pour paraphraser D. Gouadec, ont beaucoup évolué au cours des deux dernières décennies, et les interprètes sont de plus en plus sollicités pour intervenir dans des situations sociales, on parle de "community interpreting ". Cette évolution implique-t-elle une adaptation des cursus de formation, et si oui, de quel ordre?

Je ne connais pas la formule de Monsieur Daniel Gouadec (j'ai encore beaucoup à faire pour mettre la recommandation de Danica à exécution), et je ne sais pas exactement à quoi elle renvoie, sauf pour l'interprétation en langue des signes, française en l'occurrence, l'interprétation LSF. Je comprends que vous parlez par exemple de l'interprétation pour les travailleurs migrants, ou pour des personnes entendues par la justice, c'est bien cela? Ces situations se caractérisent par le déséquilibre des statuts, l'un des pôles de la communication étant socialement - statutairement - supérieur à l'autre. II en découle une polarisation des vouloirs au détriment du locuteur infériorisé. À mon avis, cela pose effectivement certains problèmes, à la fois théoriques et pratiques. Toutefois mon impression est qu'il suffirait sans doute de sensibiliser les élèves à ces situations particulières d'exercice, car le maître-mot des cursus existants à I'ESIT, en traduction comme en interprétation d'ailleurs, est déjà pour moi l'adaptation, permanente. Mais comme je n'ai été qu'exceptionnellement confronté à ce type de situation, je vous renvoie à des collègues plus compétents sur la question, comme Philippe Séro-Guillaume pour l'interprétation LSF par exemple.

- Comment voyez-vous l'avenir de la profession?

II faudrait que je retrouve ma boule de cristal, mais elle est quelque part dans un carton... Je ne prendrai pas de risque excessif en vous disant qu'il y aura toujours besoin de bons interprètes, même si l'anglais continue à se généraliser. La simultanée se répandra encore plus parce que l'équipement sera toujours plus léger. D'après ce que j'entends dire, la télé-interprétation de conférence - que j'ai un peu pratiquée chez des industriels il y a quelques années, qui s'en servaient d'ailleurs quotidiennement -, ne rencontre pas les faveurs des interprètes, ce que l'on pouvait imaginer, mais pas plus celles des chefs interprètes. Trop d'éléments paralinguistiques se perdraient à partir d'une certaine distance, quelle que soit la qualité technique de la communication. Les bons interprètes de conférence continueront donc à voyager.

- Vous pratiquez une combinaison linguistique, chinois-français, encore assez rare semblet-il, du moins vu de ce côté-ci de la planète. Quelle incidence l'éveil de la Chine peut-il avoir, selon vous, sur le marché de l'interprétation?

Le chinois-français de "conférence classique " est effectivement assez rare, et risque de le demeurer, du fait de l'expansion de l'anglais que j'évoquais à l'instant. Aujourd'hui je ne conseil- 
lerai pas à quelqu'un n'ayant que le chinois-français de se lancer dans des études d'interprète. Les conférences internationales vont se multiplier en Chine, nos collègues des autres langues commencent déjà à y aller ponctuellement, ça va continuer. La Chine c'est l'Eldorado, mais avec l'anglais. Toutefois je suis persuadé de l'intérêt pour certains couples de langues, comme le français-chinois, associés bien sûr à une bonne connaissance de l'anglais, de compétences mixtes traduction-interprétation, mais sur le terrain, pas dans les salles de conférence. Je reviens de Corée, où l'on m'a dit qu'il y aurait des besoins de ce type pour le coréenfrançais sur les vingt ou trente ans à venir, pour des entreprises coréennes travaillant en zone francophone, d'Afrique du Nord par exemple.

- Quelles sont les combinaisons linguistiques à vos yeux les plus porteuses pour les décennies à venir ?

Je ne peux pas vous répondre au-delà de ce que je viens de dire. Si vous me permettez un écart après cette longue conversation, en termes d'importance sociale, je crois que nous bénéficierons beaucoup d'une meilleure communication franco-française par exemple, une vraie communication, pas de la " com ", entre les couches dirigeantes et la population, j'ai le sentiment d'un déficit tragique, mais bon, je sors nettement du champ de votre question...

- Vous êtes passé de l'interprétation de conférence au rôle d'enseignant, y-a-t-il continuité entre ces deux professions ? Qu'est-ce que la pratique de l'interprétation apporte à l'enseignant ?

En fait j'enseigne depuis longtemps maintenant, puisque j'ai commencé dans le milieu des années 80 , quand la plupart de nos étudiants d'aujourd'hui venaient au monde. Enseigner ce que l'on pratique, là est la continuité. Et comme je n'ai jamais rien enseigné d'autre que l'interprétation ou la traduction, j'ai un peu de mal à répondre à votre seconde question, mais je dirais sans doute la faculté d'adaptation au public.

Voilà, j'ai été très honoré de votre intérêt, et vous remercie de cette interview, Freddie Plassard, je vais être obligé de me réabonner à Traduire !

Né au milieu du siècle dernier (1954) à Sedan, Gilles Ouvrard a, après un bac scientifique, appris le chinois à I'INALCO (Institut National des Langues et Civilisations Orientales) puis la traduction avec les langues français-chinois-anglais à l'ESIT (École Supérieure d'Interprètes et de Traducteurs, Traduction en 1978, Interprétation en 1980). À partir de 1980, il a mené de front à titre libéral les deux activités de traducteur et d'interprète, dans les domaines politique, économique, juridique, scientifique et technique, à la fois pour les grandes entreprises françaises qui allaient s'implanter en Chine, et dans le cadre des coopérations bilatérales publiques, notamment en tant qu'interprète officiel puis chef interprète pour le chinois auprès du Ministère des Affaires Étrangères (jusqu'en 2005). Chargé de cours puis maître de conférences associé à l'ESIT (depuis 1990), il vient de succéder récemment à Geneviève Blondy-Mauchand à la tête de la section Traduction. 